\title{
Terrylene in a 2,3-Dichloronaphthalene Crystal. New System for Optical Single-Molecule Investigations
}

\author{
M. Białkowska ${ }^{a}$, W. ChalAdaj ${ }^{b}$, A. MakARewiCz ${ }^{a}$ And B. KozAnkiewicz ${ }^{a, *}$ \\ ${ }^{a}$ Institute of Physics, Polish Academy of Sciences, al. Lotników 32/46, 02-668 Warsaw, Poland \\ ${ }^{b}$ Institute of Organic Chemistry, Polish Academy of Sciences, M. Kasprzaka 44/52, 01-224 Warsaw, Poland \\ (Received October 16, 2015) \\ Results of optical studies of a new single-molecule system, terrylene molecules in a 2,3-dichloronaphthalene \\ crystal, are presented for the first time. Analysis of the second-order fluorescence intensity correlation function \\ shows that the external heavy-atom effect (due to the chlorine atoms) contributes to the increase of the intersystem \\ crossing rate constant for the $S_{1} \rightarrow T_{1}$ transition, by one order of magnitude, whereas the rate constant for the \\ $T_{1} \rightarrow S_{0}$ transition is only weakly influenced.
}

DOI: 10.12693/APhysPolA.128.RK.128.3.1-1

PACS: 33.50.Dq, 82.37.-j

\section{Introduction}

Experiments performed at the single-molecule level, in comparison to conventional ensemble-averaging, often reveals unsuspected differences from molecule to molecule. Such investigations give access to the properties of a selected molecule in its nano-environment, and to the distribution of parameters that characterize a population.

To fulfill the requirements for single molecule optical detection the molecule has to be an efficient emitter of fluorescence, be photostable enough to emit at least thousands of photons before undergoing chemical photoreaction to a dark (non-emitting) compound. The class of molecules, and of guest-host systems, which present appropriate photophysical parameters for such studies is not very extensive. For recent reviews of the singlemolecule studies see references [1] and [2].

One of the most frequently used dye in the field of single-molecule optical studies is terrylene (Tr), which has been studied within a broad temperature range, from cryogenic to room temperature. This dye was used to probe single crystals [3-12], polymers [13-15] and Shpol'skii matrices [16-19]. In the mentioned studies, $\mathrm{Tr}$ molecules, optically excited to the $S_{1}$ state, were capable to emit more than $10^{5}$ fluorescence photons before undergoing intersystem-crossing transitions to the longlived $T_{1}$ state (in other words the triplet quantum yield was smaller than $10^{-5}$ ). A molecule in the $T_{1}$ state cannot emit fluorescence photos, thus each time spent in this state reduces the total number of emitted photons. It is well known that the rate of intersystem crossing transition increases in environments possessing heavyatoms [20], by the same talk, these media are responsible for quenching of the molecular fluorescence. It was

${ }^{*}$ corresponding author; e-mail: kozank@ifpan.edu.pl therefore surprising that $\mathrm{Tr}$ in polycrystalline ortho- [21] and para-dichlorobenzene [22], both host matrices with chlorine as the heavy-atoms, create bright and highly photostable single-molecule systems. Negligible role of the external heavy-atom effect on the Tr emission may be related to much higher triplet state depopulation rate than the rate of its population [6]. Such the interpretation was already proposed for single dibenzanthranthrene molecules isolated in frozen krypton and xenon matrices [23].

In order to get deeper insight into the role of the intersystem-crossing at the single molecule level, in the present contribution we study single $\mathrm{Tr}$ molecules dispersed in a 2,3-dichloronaphthalene (2,3-DCN) single crystal. This is a new system, which has never been studied before. The advantage of using 2,3-DCN as a host matrix is not only related to the presence of chlorine (heavy) atoms, but also to expected "dipolar" disorder of the crystal structure, where molecules are statistically aligned with their chlorine atoms in "up" or "down" directions. Such a disorder was indeed observed, and was already studied by us at the single-molecule level in $2,3-$ dimethylnaphthalene crystals $[8,11]$.

\section{Experimental}

Single crystals of zone-refined 2,3-DCN (synthesis of the compound will be provided elsewhere) lightly doped with $\operatorname{Tr}$ (purchased from the Institut für PAH-Forschung, Greifenberg, Germany) were grown by cosublimation of both components under $\approx 500$ mbar of argon (N5.0) atmosphere. Typical, high optical quality crystal "flakes" have thickness of a few tens of micrometers and a surface area of about $10 \mathrm{~mm}^{2}$.

Fluorescence and fluorescence excitation spectra of a bulk sample (at $5 \mathrm{~K}$ ) were measured with the aid of a home-built setup equipped with a liquid helium optical cryostat, McPherson 207 monochromator, EMI 9659 photomultiplier and electronic card inserted into a PC.

(RK.128.3.1-1) 
Excitation source (within 560-590 nm range) was a Coherent 700 dye laser (with the simplified optics for cw operation) pumped by a Coherent Verdi-V5 laser (532 nm).

Fluorescence decay curves were monitored with the aid of "time correlated" single photon counting technique (in inverted time mode). The system composed of a modelocked Coherent Mira-HP laser pumped by a Verdi 18 laser, an APE Pulse selector reducing repetition of a Mira laser pulses to $2 \mathrm{MHz}$, and a frequency doubling crystal. Fluorescence photons, dispersed with a McPherson 207 monochromator, were detected with a HMP-100-50 hybrid detector and a SPC-150 module inserted into a PC, both from Becker and Hickl GmbH. Fluorescence decay curves (delayed by $1 \mathrm{~ns}$ in respect of the excitation) were fitted to single exponential function without using a deconvolution procedure. Estimated precision of the decay time determination was $20 \mathrm{ps}$.

Optical experiments at the single-molecule level were done with the aid of a home-built confocal microscope $[8,9]$ equipped with a low-temperature optical cryostat, which allowed to stabilize any temperature value between 1.7 and $300 \mathrm{~K}$. Excitation source was a Coherent 700 dye + Coherent Verdi-V5 laser setup (the same as used in the bulk experiments). Excitation beam was focused on a sample to a near-diffraction-limited spot with the aid of a low-temperature microscope objective (made by Bernard Halle Nachfl. GmbH, Berlin). Fluorescence photons were collected by the same microscope objective and detected by a Perkin Elmer photon counting module SPCM-AQR 14. Residual laser light, reflected from a sample, was blocked by using appropriate set of dielectric filters (purchased from Chroma Tech. Corp.).

\section{Result and discussion}

Fluorescence and fluorescence excitation spectra of a bulk sample of $\operatorname{Tr}$ in a 2,3-DCN crystal, measured at $5 \mathrm{~K}$, are presented in Fig. 1. Maximum of the fluorescence excitation spectrum $\left(\lambda_{\mathrm{obs}}=625.9 \mathrm{~nm}\right)$ is located at $579.3 \mathrm{~nm}$ whereas maximum of the fluorescence spectrum $\left(\lambda_{\text {exc }}=532 \mathrm{~nm}\right)$ is located at $581.3 \mathrm{~nm}$. Within the scanning range of our dye laser $(560-590 \mathrm{~nm})$ the $\mathrm{flu}$ orescence excitation spectrum, with the $(0,0)$ line and the vibronic line separated from the origin by $\approx 245 \mathrm{~cm}^{-1}$, looks like a reflection image of the fluorescence spectrum.

Fluorescence decay curve for a bulk sample of $\mathrm{Tr}$ in a 2,3-DCN crystal is shown in Fig. 2. Fit to the monoexponential function is very good, with the decay time $3.47 \mathrm{~ns}$. This value is not much different from the fluorescence decay times measured for bulk samples of Tr in several solids lacking heavy atoms [24]. Such result suggests that in order to not disturb significantly the total depopulation rate of the singlet $S_{1}$ state, an increase of the $S_{1} \rightarrow T_{1}$ rate related to the heavy-atom effect cannot be higher by more than 1-3 orders of magnitude.

The confocal fluorescence image $\left(30 \times 30 \mu \mathrm{m}^{2}\right)$ of a $\mathrm{Tr} / 2,3-\mathrm{DCN}$ sample is presented in Fig. 3. Concentration of Tr molecules in this sample was sufficiently small

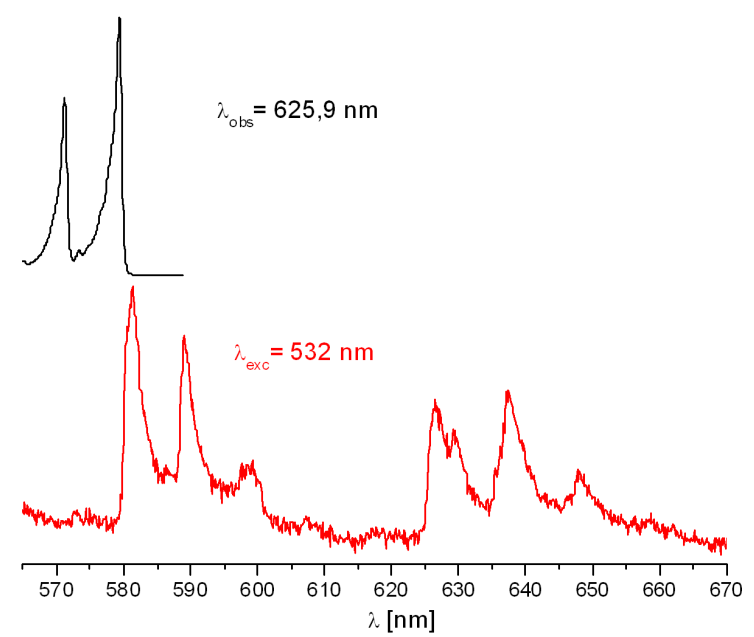

Fig. 1. Fluorescence excitation $\left(\lambda_{\text {obs }}=625.9 \mathrm{~nm}\right)$ and fluorescence $\left(\lambda_{\text {exc }}=532 \mathrm{~nm}\right)$ spectra of $\operatorname{Tr}$ in $2,3-\mathrm{DCN}$ crystal at $5 \mathrm{~K}$.

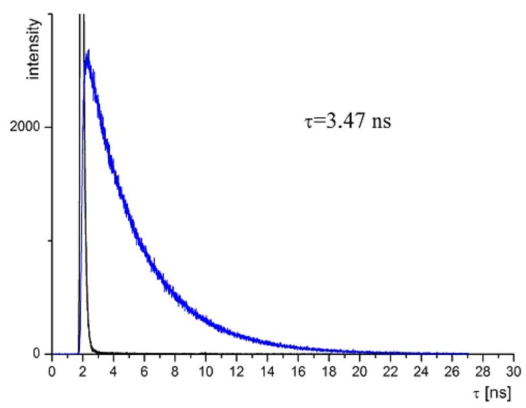

Fig. 2. Fluorescence decay (wide curve) of a bulk sample of $\operatorname{Tr}$ in a 2,3-DCN crystal at $5 \mathrm{~K}$, monitored with $\lambda_{\text {exc }}=430 \mathrm{~nm}$ and $\lambda_{\text {obs }}=581.3 \mathrm{~nm}$. Excitation pulse is shown as sharp curve.

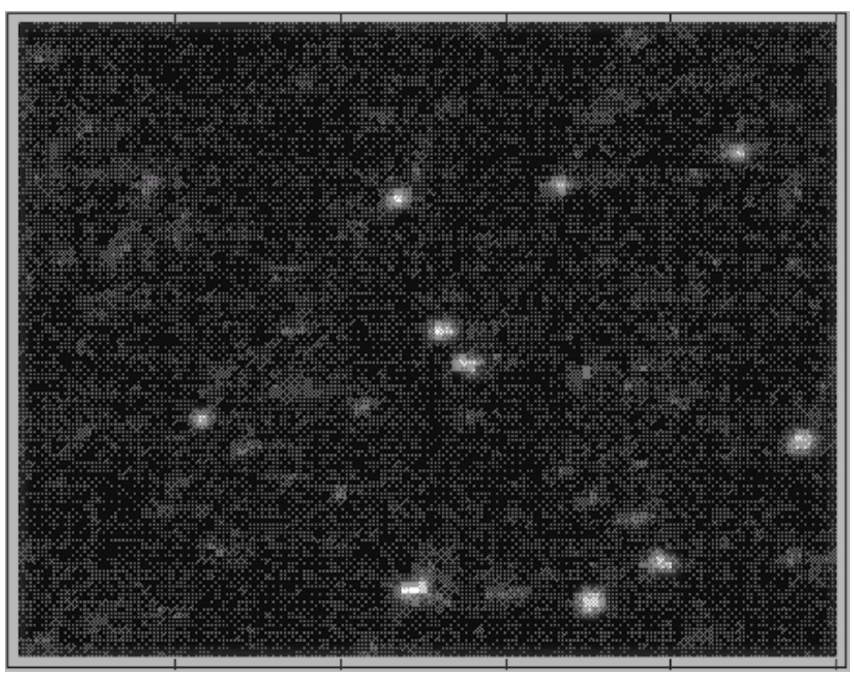

Fig. 3. Confocal fluorescence image $\left(30 \times 30 \mu \mathrm{m}^{2}\right)$ of single Tr molecules embedded in a $2,3-\mathrm{DCN}$ crystal at 5 K. $\lambda_{\text {exc }}=571.9 \mathrm{~nm}$. Bright spots correspond to single Tr molecules. 
to distinguish single molecules of $\mathrm{Tr}$ as separated bright spots. The image was obtained with the excitation wavelength of $571.9 \mathrm{~nm}$ - such light excites Tr molecules to the vibronic state located $\approx 245 \mathrm{~cm}^{-1}$ above the electronic origin. Similar confocal images for the system under investigation we were able to obtain within the whole temperature range of our setup, from the liquid helium up to the room temperature. Focusing our microscope objective on a given bright spot and collecting emitted photons we got information about a single molecule properties.

The triplet state kinetic parameters of a single molecule can be deduced from the analysis of the secondorder correlation function given by the formula [25]:

$$
g^{(2)}(\tau)=\langle I(t) I(t+\tau)\rangle /\langle I\rangle^{2},
$$

where $I(t)$ is the fluorescence photon rate at time $t$. Transition to the triplet $T_{1}$ state is a rare event and, in order to monitor any correlation between the fluorescence photon bunches, we had to collect the signal over a long period of time. A typical acquisition time for one correlation curve was $1 \mathrm{~h}$. Furthermore, to obtain information about the both rate constants, $k_{23}$ describing the $S_{1} \rightarrow T_{1}$ transition and $k_{31}$ describing depopulation of the $T_{1}$ state, for the selected single $\operatorname{Tr}$ molecule we had to collect a set of correlation functions at several excitation intensities $(I)$. Requirement of a long exposition of Tr molecules to the excitation laser light has appeared to be a real problem. Molecules were often bleached, especially at temperatures close to the room temperature and if the crystal, before being inserted into a cryostat, was stored for some hours in air atmosphere. Such behavior suggests oxidation as the primary reason of the photobleaching. We succeeded to collect sufficient sets of correlation function only for some single Tr molecules in freshly grown 2,3DCN crystals, and at temperatures below $150 \mathrm{~K}$, when diffusion of oxygen is slowed down. The set of correlation functions for a single $\mathrm{Tr}$ molecule in the 2,3-DCN crystal, collected at temperature of $125 \mathrm{~K}$ with the laser excitation intensities between 0.85 and $7 \mathrm{MW} / \mathrm{cm}^{2}$, is presented in Fig. 4.

Presented fluorescence intensity correlation functions were very well fitted with a single exponential function. Such a simple time-dependence indicates that the triplet state $T_{1}$ can be treated as a single level (at $125 \mathrm{~K}$ spin-lattice relaxation couples all three triplet spin sublevels [12]). In the formula [25]:

$$
g^{(2)}(\tau)=1+C \exp (-\tau \lambda),
$$

the contrast $C=\left(\lambda-k_{31}\right) / k_{31}$ and the decay rate $\lambda=k_{31}+k_{31} I / I_{s}\left[\left(1+k_{31} / k_{23}\right) I / I_{s}\right]^{-1}$.

Experimentally determined contrast $\left(C_{\exp }\right)$ was corrected for the background, according to the formula: $C=C_{\exp }(1+b / s)^{2}$, where $s$ and $b$ are the measured values of the signal and the background, respectively [25]. In further elaboration of the experimentally determined correlation functions we followed the procedure described already in our previous paper [6]. We found that the

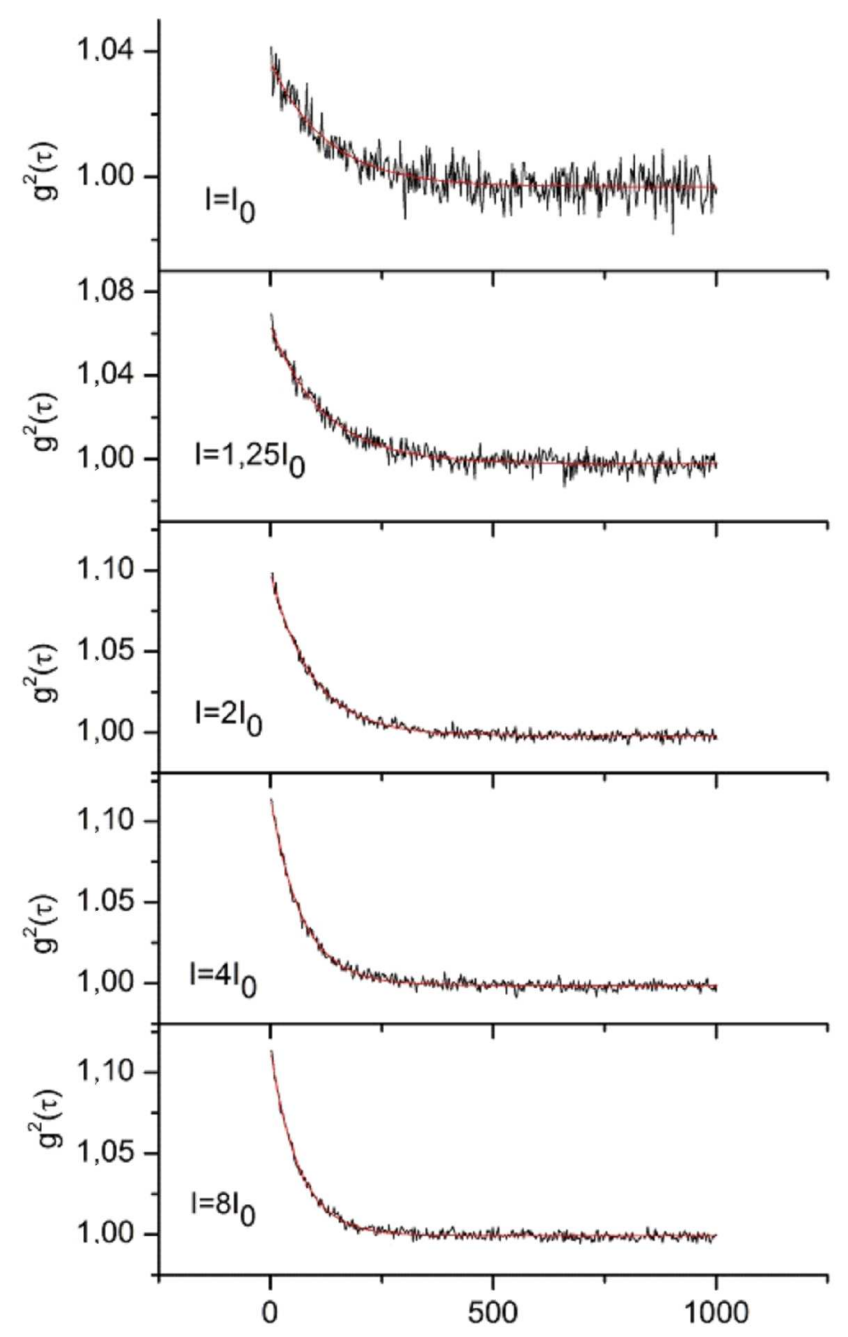

Fig. 4. Second-order fluorescence intensity correlation function of a single Tr molecule in the 2,3-DCN crystal at $125 \mathrm{~K}$, detected at different excitation laser intensities $\left(\mathrm{I}_{0}=850 \mathrm{~kW} / \mathrm{cm}^{2}\right)$.

rate constant $k_{31}$ depends linearly on the laser intensity, like it was observed in the case of $\operatorname{Tr}$ in a $p$-terphenyl crystal [6]. In the dependence

$$
k_{31}=k_{T}+\beta I \text {, }
$$

$k_{T}$ describes the intersystem crossing transition to the singlet ground state, $T_{1} \rightarrow S_{0}$, whereas $\beta I$, the triplet state depopulation by $T_{1} \rightarrow T_{n}$ absorption ( $T_{n}$ indicates higher excited triplet state) followed by $T_{n} \rightarrow S_{1}$ intersystem crossing. For the molecule contributing to Fig. 4 we found that

$$
k_{T}=(6.0 \pm 0.4) \times 10^{3}\left[\mathrm{~s}^{-1}\right]
$$

and the slope

$$
\beta=0.9\left[\mathrm{~cm}^{2} /(\mathrm{kW} \mathrm{s})\right] \text {. }
$$

Next, plotting $\left(\lambda-k_{31}\right)$ as a function of the laser-light intensity we found that

$$
k_{23}=(16.2 \pm 4.9) \times 10^{3}\left[\mathrm{~s}^{-1}\right] .
$$


Similar analysis was done for some other Tr molecules in the 2,3-DCN crystal. Although each single molecule differs from the others due to a different environment, and the rate constants are estimated with significant errors, comparing Tr molecules in a 2,3-DCN crystal with the situation of $\operatorname{Tr}$ molecules in a $p$-terpenyl crystal [6] we can generally conclude that:

1. The rate constant $k_{T}$ (describing the $T_{1} \rightarrow S_{0}$ intersystem crossing transition) is only slightly disturbed by the external heavy-atom effect (it is $\approx 50 \%$ higher for the above considered Tr molecule at $125 \mathrm{~K}$ );

2. The rate constant $k_{23}$ (describing the $S_{1} \rightarrow T_{1}$ intersystem crossing transition) is usually about 1 order of magnitude higher for the studied system, indicating stronger heavy-atom effect on this transition.

Such a situation can be rationalized if one inspects the energy diagram of the electronic states of Tr. According to quantum chemistry calculations [26] the triplet $T_{1}$ state of $\mathrm{Tr}$ is located about $1 \mathrm{eV}$ above the $S_{0}$, and very far, ca. $1.2 \mathrm{eV}$, below the lowest excited singlet state $S_{1}$. The mechanism responsible for the external heavy-atom effect is the spin-orbit coupling [20], and the coupling of triplet and singlet states depends reversibly on the energy difference between the states. Deep energy location of the $T_{1}$ state prevents efficient admixture of the singlet character to this state, by the same $T_{1} \rightarrow S_{0}$ should be not (or only weakly) sensitive to external heavy-atom effect. Situation is different for the $S_{1} \rightarrow T_{1}$ transition. At the energy slightly above the singlet $S_{1}$ state there are other singlet and triplet states (of appropriate symmetry) which can be coupled to each other by the spin-orbit interaction.

The simple explanation presented above needs further theoretical verification, and this work is in progress. We are also on the way to synthesize 2,3dibromonaphthalene, which would provide a crystalline matrix with a stronger heavy-atom effect.

Finally, let us mention that the small increase of the triplet yield for $\mathrm{Tr}$, from $10^{-5}$ in a $p$-terphenyl crystal to $10^{-4}$ in a $2.3-\mathrm{DCN}$ crystal, is not able to contribute to noticeable shortening of the fluorescence decay time, as we observed for the bulk sample.

\section{Acknowledgments}

The work was partly financially supported by the grant no. 2012/04/A/ST2/00100 of the Polish National Science Centre.

\section{References}

[1] W.E. Moerner, Angew. Chem. Int. Ed. 54, 8067 (2015).

[2] B. Kozankiewicz, M. Orrit, Chem. Soc. Rev. 43, 1029 (2014).
[3] S. Kummer, F. Kulzer, R. Kettner, T. Basche, C. Tietz, C. Glowatz, C. Kryschi, J. Chem. Phys. 107, 7673 (1997).

[4] L. Fleury, B. Sick, G. Zumofen, B. Hecht, U.P. Wild, Mol. Phys. 95, 1333 (1998).

[5] T. Nonn, T. Plakhotnik, Chem. Phys. Lett. 336, 97 (2001).

[6] M. Banasiewicz, O. Morawski, D. Wiącek, B. Kozankiewicz, Chem. Phys. Lett. 414, 374 (2005).

[7] A. Nicolet, M. Kol'chenko, B. Kozankiewicz, M. Orrit, J. Chem. Phys. 124, 164711 (2006).

[8] M. Banasiewicz, D. Wiącek, B. Kozankiewicz, Chem. Phys. Lett. 425, 94 (2006).

[9] M. Banasiewicz, J. Dresner, O. Morawski, D. Wiącek, B. Kozankiewicz, Acta Phys. Pol. A 112, S85 (2007).

[10] D. Wiącek, B. Kozankiewicz, Chem. Phys. Lett. 462, 280 (2008).

[11] R. Kościesza, E. Luzina, D. Wiącek, J. Dresner, B. Kozankiewicz, Mol. Phys. 107, 1889 (2009).

[12] M. Białkowska, A. Makarewicz, M. Banasiewicz, B. Kozankiewicz, Chem. Phys. Lett. 555, 131 (2013).

[13] M. Orrit, J. Bernard, A. Zumbusch, R.I. Personov, Chem. Phys. Lett. 196, 595 (1992).

[14] B. Kozankiewicz, J. Bernard, M. Orrit, J. Chem. Phys. 101, 9377 (1994).

[15] A.C. Wirtz, M. Dokter, C. Hofman, E.J.J. Groenen, Chem. Phys. Lett. 417, 383 (2006).

[16] W.E. Moerner, T. Plakhotnik, T. Irngartinger, M. Croci, V. Palm, U.P. Wild, J. Phys. Chem. 98, 7382 (1994).

[17] M. Vacha, Y. Liu, H. Nakatsuka, T. Tani, J. Chem. Phys. 106, 8324 (1997).

[18] M. Matsushita, A. Bloess, Y. Durand, J.Y.P. Butter, J. Schmidt, E.J.J. Groenen, J. Chem. Phys. 117, 3383 (2002).

[19] M. Banasiewicz, D. Wiącek, B. Kozankiewicz, Chem. Phys. Lett. 425, 289 (2006).

[20] S.P. McGlynn, T. Azumi, M. Kinoshita, Molecular Spectroscopy of the Triplet State, Prentice-Hall, Englewood Cliffs, New Jersey 1969.

[21] A.A. Gorshelev, A.V. Naumov, I.Y. Eremchev, Y.G. Vainer, L. Kador, J. Köhler, Chem. Phys. Chem. 11, 182 (2010).

[22] N. Navarro, Y. Tian, M. van Stee, M. Orrit, Chem. Phys. Chem. 15, 3032 (2014).

[23] J. Sepioł, A. Starukhin, R. Kołos, T. Latychevskaia, J. Jasny, A. Renn, U.P. Wild, Chem. Phys. Lett. 311, 29 (1999).

[24] G.S. Harms, Th. Irngartinger, D. Reiss, A. Renn, U.P. Wild, Chem. Phys. Lett. 313, 533 (1999).

[25] J. Bernard, L. Fleury, H. Talon, M. Orrit, J. Chem. Phys. 98, 850 (1993).

[26] I. Deperasińska, E. Karpiuk, M. Banasiewicz, B. Kozankiewicz, Chem. Phys. Lett. 492, 93 (2010). 